sudden onset, oedema of retinal tissues, equally sudden cessation on removal of the cause and complete restoration of vision.

(2) Whenever chronic nephritis complicates pregnancy, the visual disturbances are due to renal retinitis.

(3) This retinitis is associated with high blood-pressure which, together with the signs of retinitis, have a tendency to persist after delivery.

(4) This form of retinitis forms a useful guide in differentiating chronic nephritis complicating pregnancy from pre-eclamptic toxaemia.

(5) It should really belong to the group of cases having hypertension and chronic nephritis preceding pregnancy.

(6) Apart from their differentiating value, lesions of the retina help us to estimate the damage done to the vascular tree.

(7) In the presence of commencing retinitis, the ophthalmologist should not hesitate to advise interruption of pregnancy.

\title{
REFERENCES
}

1. Foster Moore, R.- "Medical Ophthalmology." Second edition. pp. 175-176.

2. Williams, J. Whitridge.- "Obstetrics." Sixth edition, pp. 662, 635-651.

3. Wagener, Henry P.-Amer. Jl. of Ophthal., Vol. XVIII, pp. 263-264, 1935.

\section{THE RECOGNITION OF FLASHING COLOURED LIGHTS BY PERSONS WITH NORMAL AND DEFECTIVE COLOUR VISION}

BY

\section{H. V. Corbett and H. E. ROAF}

(FROM THE DEPARTMENT OF PHYSIOLOGY, THE UNIVERSITY OF LIVERPOOL)

Flashing lights and stationary lights viewed from a tossing vessel may be visible for only a short interval of time at each exposure, therefore it is important in testing colour vision to vary the duration of the exposure. Coloured lights are the forms of stimuli which occur in those trades in which colour recognition is important, for example, in railway, marine and air services; therefore the recognition of coloured lights is the most practical form of test. Recognition of coloured lights does not require training, whilst viewing objects through a telescope requires practice.

The Edridge-Green Lantern has apertures of different areas which when viewed at a constant distance of 20 feet represent fixed lights (e.g., a railway " bull's eye ") at different distances.

In 1912 a committee recommended a lantern for use by the Board of Trade. This lantern, in addition to a large single aperture, can be used with two small apertures, both of which may 
show the same or different colours. The main objection to the Board of Trade Lantern is that the colours are said to be of equal luminosity. This comparison of luminosity is apparently made by a normal individual and there is no guarantee that they would appear of equal brightness to subjects with defective colour vision, hence the lights might be distinguished by their unequal brightness. Pitt (1935, p. 32) mentions the use of neutral filters to alter the brightness as desired. We also have made use of neutral filters throughout our work.

The work described below formed part of that done for a thesis for the degree of Doctor of Medicine of the University of Liverpool (Corbett, 1934).

\section{The Lantern}

The lantern constructed for this work, like that recommended by the Board of Trade Committee, could show a single large aperture or two smaller apertures. The single large aperture $(0.2$ inches in diameter) when viewed at a distance of 20 feet represents a 6 inch aperture 200 yards away. The two smaller apertures ( 0.02 inches in diameter), 1 inch apart, represent two 6 inch apertures 25 feet apart at a distance of 2,000 yards when viewed at a distance of 20 feet. The colours, which were $W$ ratten filters, were duplicates and were selected with the object of finding out what selection of wave-lengths would be most confusing to those with
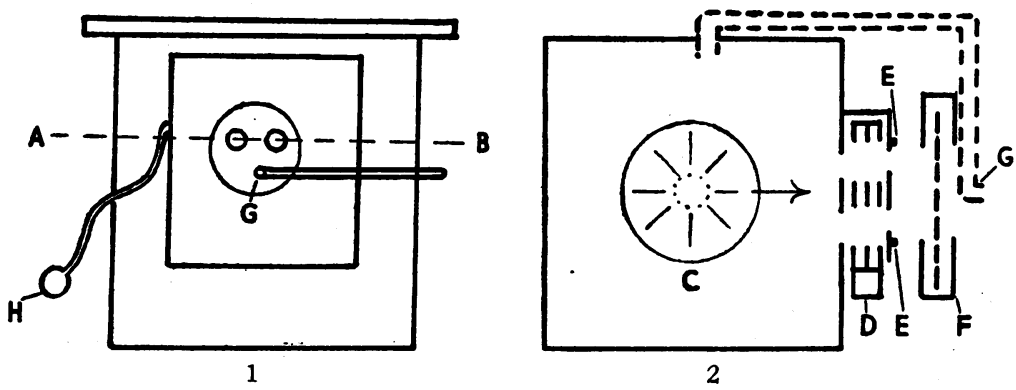

Diagram of Colour Testing Lantern.

1. Front elevation looking through the Thornton-Pickard shutter to see the two small apertures in the brass plate and the fixation light $G$ in front of the shutter. $H$ is the bulb for operating the shutter.

2. Section through plane $\mathrm{A}-\mathrm{B}$ showing the light, $\mathrm{C}$; the slider, $D$, with slots for holding coloured screens and neutral glasses; the studs E, E, on which the brass plates can be hung so as to allow either one large or two small apertures to be exposed and the ThorntonPickard shutter, F, for regulating the duration of the exposure. The glass rod, $G$, for the fixation light is shown by interrupted lines to indicate that it is below the level of the section, thercfore it does not interfere with the view of the hole in the brass plate which really lies above the rod. 
defective colour vision. By the use of neutral filters two identical wave-length compositions could be shown with different intensities of $\frac{1}{2}, \frac{1}{4}, 1 / 10$ or $1 / 100$ of their original intensity. Many different mechanical means might be used for obtaining the same results, but the actual lantern used consisted of a gas filled opal electric light bulb inside a light-tight box. Two holes in the front of the box allowed light to pass out towards the subject. On the front of the box were two studs on which one of two metal plates could be placed. One plate had a single aperture 0.2 inches in diameter opposite one of the holes in the box. This allowed a single large light to be seen, the other hole being obscured by the metal plate. The other plate had two holes 0.02 inches in diameter one inch between the centres so that two small lights were visible, one corresponding to each of the holes in the box. A carrier with compartments could be slid into place between the holes in the box and the metal plate, so that various filters could be shown and the intensity modified by neutral filters in series with the coloured filters.

To vary the duration of exposure of the lights, a ThorntonPickard shutter was mounted in front of this lantern.

In order that the subject should look in the right direction a weak fixation light was used. This consisted of a glass rod 10 inches long, one end of which was fixed into the side of the lantern, bent round so that the other end faced towards the subject. Except for the end of this rod, facing the subject, the whole was suitably screened to prevent the escape of stray light. By internal reflection a certain amount of light passed out at the exposed end which was ground to a fairly fine finish. This light was not strong enough to dazzle the subject, therefore it did not interfere with the observation of the coloured lights, but it ensured that the subject was looking in the right direction when the exposure was made.

Three speeds of shutter were used, $0.0218,0.0288$ and 0.0350 seconds, and any longer period of one second or more could be used by keeping the operating bulb pressed when the shutter was set for time exposures.

\section{Methods of Examination}

By the lantern described above one can expose either one large light which can be varied in colour and in intensity or two small lights which may be $(a)$ identical in colour and intensity, $(b)$ of the same colour but of different intensities, $(c)$ similar but of different wave-length composition, $(d)$ entirely different lights. The duration of exposure of any of these can be varied.

The subject looked at the lantern from a standard distance of 20 feet. 
The cases examined were also tested by the Ishihara pseudoisochromatic plates and the Nagel Anomaloscope. The former is a quick and convenient method for detecting defects in colour vision, the latter is also a good test for defective colour vision but training is necessary to recognise the fields of view through the telescope (Hailwood and Roaf, 1937).

\section{Results}

The results consist of detailed records of naming colours by normal and by hypochromatic individuals. The tables showing the results would occupy too much space to print them in detail.

\section{Discussion}

Normal Subjects.- Normal persons do not make many mistakes in colour recognition. Some of them have difficulty in recognising red No. 70 which furnishes a dim light from the extreme long wave-length end of the visible spectrum. Mistakes are sometimes made in naming colours in the blue-green series, but we do not think that the latter region is of practical importance. The former mistake is less noticeable after dark adaptation and when the accompanying light is not too bright. The nomenclature of blue and of green seems to vary considerably and it is markedly dependent upon the state of adaptation. The distinction between blue and green is not of importance for marine and other signals.

Some of the normal persons can recognise colours with the shortest duration used (0.0218 seconds). These individuals might be selected for special purposes such as "look out " men in the navy (1933, p. 29). Most normal persons tend to make mistakes when the duration is less than 0.0350 seconds.

Subjects with defective Colour Vision.-Subjects afflicted with defective colour vision are more influenced by the duration of exposure than are normal people. It is possible to divide them into three arbitrary types. We do not pretend that there is a hard and fast division between the groups but the division is useful as a means of classification.

Type I: Those who are liable to confuse red and green even with the large aperture (0.2 inch at 20 feet) and with long durations of exposure.

Type II : These subjects can generally distinguish the colours with the large aperture but their accuracy diminishes with the smaller apertures ( 0.02 inch at 20 feet) and when the duration of exposure is decreased to $1 \mathrm{sec}$. or less. However, when the exposure lasts 1 second or more the mistakes with the large aperture are not so frequent and improvement in the recognition of the colours is shown also with the smaller apertures. 
Type III : These subjects are frequently unaware of their defect until specially examined and they make no mistakes with the large aperture with all durations used. With the small apertures they immediately manifest their defect when the durations are less than 0.0350 seconds. Durations of 1 second or more enable them to make more correct answers in nearly all cases.

This type would pass a perfect lantern test if given an " unlimited" duration of exposure of perhaps a few seconds but they might show their defect by the Ishihara plates. They would be unsafe for the recognition of coloured lights of the " intermittent flashing " variety especially at great distances unless the exposure lasted 1 second or more.

These results were obtained after at least 15 minutes dark adaptation. Without dark adaptation the colour recognition is slightly worse in all cases.

Summary.-Our results support those of Guttmann $(1907,1908)$ in showing that the person with defective colour vision is more dependent than the normal subject upon

(a) the size of aperture, i.e., the angular aperture of a coloured light ;

(b) the brightness of the lights, and

(c) the duration of exposure.

It would seem that there are gradations in the degree of the defect so that one can recognise many degrees of defective colour vision. By the hypochromat red is more frequently named correctly than is green. This may be accounted for by the fact that the latter is less saturated or in other words it is not so far removed from parts of the spectrum which give rise to the blue sensation : the blue sensation being complementary to the second sensation of the dichromat (see Pitt, 1935).

\section{REFERENCES}

Board of Trade (1912).--Report of the Departmental Committee on Sight Tests, pp. 15-16. Cd. 6256. His Majesty's Stationery Office, London.

Corbett, H. V. (1934).-Studies in Colour Discrimination. The Library, The University of Liverpool.

Guttmann, A. (1907).-Zeitschr. f. Psychol. d. Sinnesorg., Vol. XLII, pp 24 and 250 .

Tail (1908).-Zeitschr.f. Psychol. d. Sinnesorg., Vol. XLIII, pp. 146, 199, 255.

Hailwood, J. G. and Roaf, H. E. (1937). - Jl. of Physiol. In the press.

Medical Research Council (1933). - Colour Vision Requirements in the Royal Navy. His Majesty's Stationery Office, London.

Pitt, F. H. G. (1935). Characteristics of Dichromatic Vision. His Majesty's Stationery Office, London. 